\title{
Preliminary laboratory studies of the optical scattering properties of the crystal clouds
}

\author{
C. Saunders ${ }^{1}$, J. Rimmer ${ }^{1}$, P. Jonas ${ }^{1}$, J. Arathoon ${ }^{1}$, C. Liu ${ }^{2}$ \\ ${ }_{1}^{1}$ Physics Department, University of Manchester Institute of Science and Technology, Sackville Street, Manchester, M60 1QD, UK \\ 2 Department of Meteorology, The University of Reading, Earley Gate, PO Box 243, Reading, RG6 6BB, UK
}

Received: 30 April 1997 / Revised: 12 November 1997 / Accepted: 24 November 1997

\begin{abstract}
Ice crystal clouds have an influence on the radiative budget of the earth; however, the exact size and nature of this influence has yet to be determined. A laboratory cloud chamber experiment has been set up to provide data on the optical scattering behaviour of ice crystals at a visible wavelength in order to gain information which can be used in climate models concerning the radiative characteristics of cirrus clouds. A PMS grey-scale probe is used to monitor simultaneously the cloud microphysical properties in order to correlate these closely with the observed radiative properties. Preliminary results show that ice crystals scatter considerably more at $90^{\circ}$ than do water droplets, and that the halo effects are visible in a laboratorygenerated cloud when the ice crystal concentration is sufficiently small to prevent masking from multiple scattering.
\end{abstract}

Key words. Meteorology and atmosphere dynamics . Climatology $\cdot$ Radiative process $\cdot$ Atmospheric composition and structure $\cdot$ Cloud physics and chemistry

\section{Introduction}

Clouds form throughout the depth of the troposphere, where they play a major role in the earth's radiation balance and so influence weather and climate. Solar radiation reaching the top of the atmosphere has approximately $9 \%$ of its energy in wavelengths shorter than the visible, $40 \%$ in the visible region $(0.4-0.7 \mu \mathrm{m})$ and about $51 \%$ in longer wavelengths up to about $5 \mu \mathrm{m}$ (Monteith and Unsworth, 1990). Although significant

Correspondence to: C. P. R. Saunders

Fax: 44161200 3941; e-mail: clive.saunders@umist.ac.uk progress in recent years has been made in understanding the radiative properties of water clouds, our knowledge of both the physical properties and the radiative properties of ice clouds is still fairly fragmentary. A knowledge and physical understanding of the scattering and absorption properties of atmospheric ice in the solar and infrared spectral bands is of great importance to studies in key areas of meteorological research. The ability to use active and passive remote-sensing techniques to determine both the macroscopic and microscopic properties of ice clouds would be enhanced if the relationships between their optical properties, scattering and absorption and general physical properties were known with greater certainty.

Dowling and Radke (1990) have listed typical values of the physical properties of cirrus clouds, Table 1 . They note that there does not appear to be any information as to the relative abundance of cirrus over any large geographical area, or at any single place for any significant length of time. Their review does not include information on the radiative properties of cirrus. They emphasise the uncertainty in determining the number density of ice crystals smaller than can be measured using currently available airborne instruments, which is below about $50-70 \mu \mathrm{m}$ in size.

Linking the physical properties and the optical properties of the ice crystal clouds is an important problem that has yet to be satisfactorily solved. In particular, some workers have attributed significant radiative effects to the small particles present, which makes the real situation even harder to unravel. Recent measurements of cirrus from ground-based observations have been correlated with satellite data by Brogniez et al. (1995); cloud optical depth and the angular scattering effect of the crystals were determined but assumptions had to be made concerning the crystal habit and orientation.

Liou $(1980,1981)$ give a general introduction to the field of atmospheric radiation and clouds. A parallel beam of radiation passing through a medium will be attenuated by its interaction with matter. If the initial intensity of the radiation is $I_{\lambda}$ and this changes to 
Table 1. Physical properties of cirrus (Dowling and Radke, 1990)

\begin{tabular}{lll}
\hline & typical value & range \\
\hline cloud centre altitude & $9 \mathrm{~km}$ & $4-20 \mathrm{~km}$ \\
cloud thickness & $1.5 \mathrm{~km}$ & $0.1-8 \mathrm{~km}$ \\
crystal number density & $30 \mathrm{~L}^{-1}$ & $10^{-4}-10^{4} \mathrm{~L}^{-1}$ \\
ice water content & $0.025 \mathrm{~g} \mathrm{~m}^{-3}$ & $10^{-4}-1.2 \mathrm{~g} \mathrm{~m}^{-3}$ \\
crystal size & $250 \mu \mathrm{m}$ & $1-8000 \mu \mathrm{m}$ \\
\hline
\end{tabular}

$I_{\lambda}+d I_{\lambda}$ after passing through a thickness $d s$ in the direction of propagation, then

$\mathrm{d} I_{\lambda}=-k_{\lambda} \rho I_{\lambda} \mathrm{d} s$,

where $\rho$ is the density of the medium and $k_{\lambda}$ denotes the mass extinction coefficient (in units of area per unit mass) for radiation of wavelength $\lambda$. The mass extinction cross-section is the sum of the mass absorption and scattering cross-sections. Thus the reduction in intensity is caused by absorption in the material as well as by scattering of radiation by the material.

The intensity may be strengthened by emission from the material plus multiple scattering from all other directions into the parallel beam, at the same wavelength. It is usual to define the source coefficient $j_{\lambda}$ such that the increase of intensity due to emission and multiple scattering is given by

$\mathrm{d} I_{\lambda}=j_{\lambda} \rho \mathrm{d} s$,

where $j_{\lambda}$ has the same physical meaning as the mass extinction cross-section. Upon combining Eqs. (1) and (2) we obtain

$\mathrm{d} I_{\lambda}=-k_{\lambda} \rho I_{\lambda} \mathrm{d} s+j_{\lambda} \rho \mathrm{d} s$.

Also it is convenient to define the source function $J_{\lambda}$ such that

$J_{\lambda} \equiv j_{\lambda} / k_{\lambda}$.

In this manner, the source function has units of radiant intensity. It follows that Eq. (3) may be rearranged to yield

$\frac{\mathrm{d} I_{\lambda}}{k_{\lambda} \rho \mathrm{d} s}=-I_{\lambda}+J_{\lambda}$.

This is the general equation of transfer without any coordinate system imposed.

Theoretical calculations of absorption, transmission, reflection, emission and scattering of electromagnetic radiation in ice (or water) require knowledge of the laboratory measurements of the complex refractive index of ice (or water) as a function of wavelength (Warren, 1984). Between about 0.2 and $5 \mu \mathrm{m}$, the optical properties of ice and water are very similar, with similar real and complex indices of refraction. The real indices show a large increase at a wavelength around $3 \mu \mathrm{m}$, while the imaginary indices show that both ice and water are highly transparent in the visible with a minimum value at around $0.46 \mu \mathrm{m}$. The imaginary index generally increases in this spectral window, with a few minor absorption bands and one major one at $3 \mu \mathrm{m}$, which is due to vibrational modes involving the stretching of the $\mathrm{O}-\mathrm{H}$ covalent bond (Warren, 1984).

Many workers have contributed to the measurement of the optical properties of ice and water and the results of these studies have been compiled in several review papers; Warren (1984) covers the optical properties of ice, Hale and Querry (1973) cover water, and Downing and Williams (1975) cover the optical properties of water in the infrared only.

The first experimental studies on the angular scattering properties of artificially generated ice crystals were by Huffman and Thursby (1969). They made angular scattering measurements using a single detector within the angular range $10^{\circ}-150^{\circ}$ using a zirconium light source with a wavelength of $0.55 \mu \mathrm{m}$. Light scattering measurements have also been made by Huffman (1970), Dugin et al. (1971, 1977), Liou et al. (1976), Sassen and Liou (1979a, b) and Pluchino (1987). Sassen and Liou (1979a, b) created a dense cloud of very small crystals with sizes normally less than $50 \mu \mathrm{m}$ and measured the scattered light from many crystals at once. Another approach is to measure the scattering properties of one crystal at a time. This method is suitable for measuring the scattering properties of much larger crystals, although the technique requires a large number of detectors to measure the angular scattering pattern for each particle that passes through the sample volume. Pluchino (1987) used an array of detectors in conjunction with a quadrupole levitator to electrodynamically suspend both ice crystals and water drops within the scattering sample volume. He measured the angular scattering patterns for single ice crystals of about $100 \mu \mathrm{m}$ using an incident wavelength of approximately $1 \mu \mathrm{m}$.

To date, measurements have mainly been taken for smaller crystals, for example Huffman and Thursby (1969) and Huffman (1970) used ice crystals with modal diameters of up to $25 \mu \mathrm{m}$. Other reported measurements such as Dugin et al. (1977) and Sassen and Liou (1979a, b) never used ice crystals exceeding $100 \mu \mathrm{m}$. Although cirrus ice crystals may grow up to $2 \mathrm{~mm}$ in size, the larger ice crystals have much lower number densities than the smaller ice crystals, but the particle size range which is most responsible for cirrus light scattering at any given wavelength has yet to be determined.

Laboratory scattering experiments have confirmed that ice crystals scatter more light than spherical water drops around the $90^{\circ}$ scattering angle, and less light in the forward and backward directions; Sassen and Liou (1979a, b) presented angular scattering intensities in terms of a normalised phase function. Anisotropic scattering effects of ice crystals were studied by Dugin et al. (1977) who made measurements in a number of azimuthal planes, scattering phase functions being presented for $0^{\circ}$ (horizontal), $30^{\circ}, 60^{\circ}$ and $90^{\circ}$ scattering planes. The results showed that the scattering phase functions varied from one azimuthal angle to another; however, it is not clear whether the variability in azimuthal directions is due to significant variation in particle shape or is caused by the anisotropic effect of 
the sample of ice crystals (Liou, 1981). Thus further investigation is required into the anisotropic scattering effects of ice crystals.

Bunting (1980) gives a short review on sensing ice clouds from satellites and Liou (1981), in his review on the optical properties of ice clouds, devotes some time to remote-sensing techniques. Satellite radiation measurements have been made in various spectral channels in the visible, near-infrared, infrared and microwave regions. Bunting (1980) concludes that our knowledge of the scattering properties of ice clouds is not yet good enough to allow satellite radiation observations to be related accurately to in situ cloud particle measurements.

Aircraft can make both microphysical and optical cloud measurements, see for example Paltridge and Platt (1981) and Foot (1988). One of the major difficulties with in situ microphysical measurements has been that methods of observing small particles, below $50 \mu \mathrm{m}$ or so, have been limited to using either a Forward Scattering Spectrometer Probe (FSSP) or a formvar replicator. The FSSP tends to be highly unreliable for accurate counting of small ice crystals, especially in the presence of large irregular ice crystals and for high ice clouds, while the replicator generally has too low a sample volume to obtain accurate size spectra. Platt et al. (1989) give evidence for significant numbers of ice crystals in the cirrus clouds studied, smaller than $70 \mu \mathrm{m}$, which was the cut-off point for their 2D imaging probe. Radiative measurements made from aircraft tend to be in the visible, near-infrared and infrared spectral regions, and are often broad-band measurements. Small-scale angular scattering measurements have not yet been made from aircraft because of experimental difficulties; however, by flying the plane in a banked orbit below a cirrus layer and measuring transmittance at $1.25 \mu \mathrm{m}$, Foot (1988) was able to construct a partial scattering phase function over the range $30^{\circ}-87^{\circ}$. Recently, Gayet et al. (1997) have described a polar nephelometer for airborne use which will provide scattering data from ice crystal clouds.

Lidar (light detection and ranging) employs the same back-scattering principle as microwave radar. Techniques have been developed to map and track concentrations of particulate matter, to study the density profile of the atmosphere, and cloud compositions. Further developments have included the use of multiple wavelength lidars for determining the minor gaseous composition by means of differential absorption techniques, the use of Doppler techniques for determining the motion of particles and molecules, and the use of the Raman scattering technique in which weak scattering occurs at shifted wavelength for water vapour measurements (Liou, 1980).

Major field experiments on the optical properties of ice clouds which have combined in situ (airborne and surface based) measurements with satellite data, have been made in recent years. They include the First ISCCP Regional Experiment (FIRE), FIRE II and the International Cirrus Experiment (ICE). Analysis of these projects has been presented by Kinne et al. (1992),
Arnott et al. (1994) and Brogniez et al. (1995), among others.

Modelling work in atmospheric radiation studies is being undertaken on various scales, from the microscopic through mesoscale and synoptic to full globalscale models. Generally the approach taken and the complexity of these models varies enormously. Many models describe the optical properties of clouds in an isolated manner, while others try to link cloud optical properties with cloud dynamics and atmospheric motions. For the more complicated models, including general circulation and climate models, exact methods for calculating cloud radiative transfer will be abandoned in favour of less time-consuming parameterisation methods (Kinne, 1987). Microphysical scattering models for ice crystal clouds have been developed since the 1960s. Early models tried to simplify ice crystal modelling by using ice sphere, ice cylinder and ice spheroid approximations, which are described in detail by Liou (1981). None of these are entirely satisfactory and will not generate the halo features that are typical of hexagonal columns and plates.

In order to model the light scattering properties of hexagonal ice crystals more exactly, geometrical ray tracing has to be employed. Angular scattering patterns were first reported by Jacobowitz (1970), while further progress has been outlined by Liou (1981). Recent developments in modelling have been made by Takano and Liou (1989a, b) taking into account the birefingence properties of ice and the multiple scattering of ice clouds. Most recently, Liu et al. (1995, 1996a, b) have extended the ray-tracing techniques to determine phase functions for single hexagonal bullet-like ice crystals and other crystal shapes. Mischenko et al. (1996) reported that ice crystal shape is crucially important to accurate determination of cloud optical properties, a result which confirms the need for laboratory studies using real crystals.

\section{Experimental apparatus and measurement techniques}

An ice crystal cloud is grown inside a laboratory cold room having internal dimensions of approximately $2 \mathrm{~m} \times 2 \mathrm{~m} \times 2 \mathrm{~m}$ with insulated walls $0.2 \mathrm{~m}$ thick. The temperature in the cold room can be reduced to a minimum of $-40{ }^{\circ} \mathrm{C}$ under thermostatic control; a double-door airlock helps to reduce heat exchange when entering. A small cloud chamber with a floor area of $1.2 \mathrm{~m} \times 1.2 \mathrm{~m}$ and a height of $0.9 \mathrm{~m}$ has been constructed inside the cold room; the chamber walls are painted matt black in order to reduce reflections. A boiler is used to create the water droplet cloud inside the cloud chamber from which the ice cloud can be produced by a suitable cloud seeding nucleation technique.

Inside the cold room, there is space for a PMS-2DGrey ice crystal sampling probe which has been positioned below the chamber to provide ice crystal particle size and concentration measurements. An air pump draws the cloud down through a hole in the base 
of the chamber to pass through the $2 \mathrm{D}$ probe sample volume for particle imaging to take place. An FSSP could be positioned in a similar manner when water cloud measurements were required. Figure 1 shows the experimental set-up. The scattering chamber is placed on the floor of the cloud chamber above the $2 \mathrm{D}$ probe so that both the light scattering measurements and the particle size and concentration measurements are made on the same volume of ice crystals. A weak updraught can be created above the scattering chamber in order to extend the growth time and hence the size spectrum of the crystals in the chamber.

An ice crystal cloud was produced from a supercooled water droplet cloud by artificial nucleation by inserting a liquid-nitrogen-cooled rod into the cloud to create a large quantity of small ice crystals that grow in the presence of the supercooled water drops and saturated vapour. The largest ice crystals that can be grown easily in the cold room in this way are about $120 \mu \mathrm{m}$ in size; using levitation techniques (by gently drawing air out of the cloud chamber through the roof) crystals of a few hundred micrometers have been observed at temperatures around $-30{ }^{\circ} \mathrm{C}$, but with special techniques and a larger cold room, ice crystals up to $800 \mu \mathrm{m}$ may be achieved. The theory of ice crystal growth has been described by, amongst others, Mason (1971) and Hobbs (1974), and the classic paper on ice crystal habit classification as a function of temperature and supersaturation is by Magono and Lee (1966). The habits of the ice crystals grown in the chamber follow the established temperature and supersaturation dependence discussed by Magono and Lee. Ice crystal observations in cirrus clouds by Arnott et al. (1994) have shown a size range from 20 to $500 \mu \mathrm{m}$; they

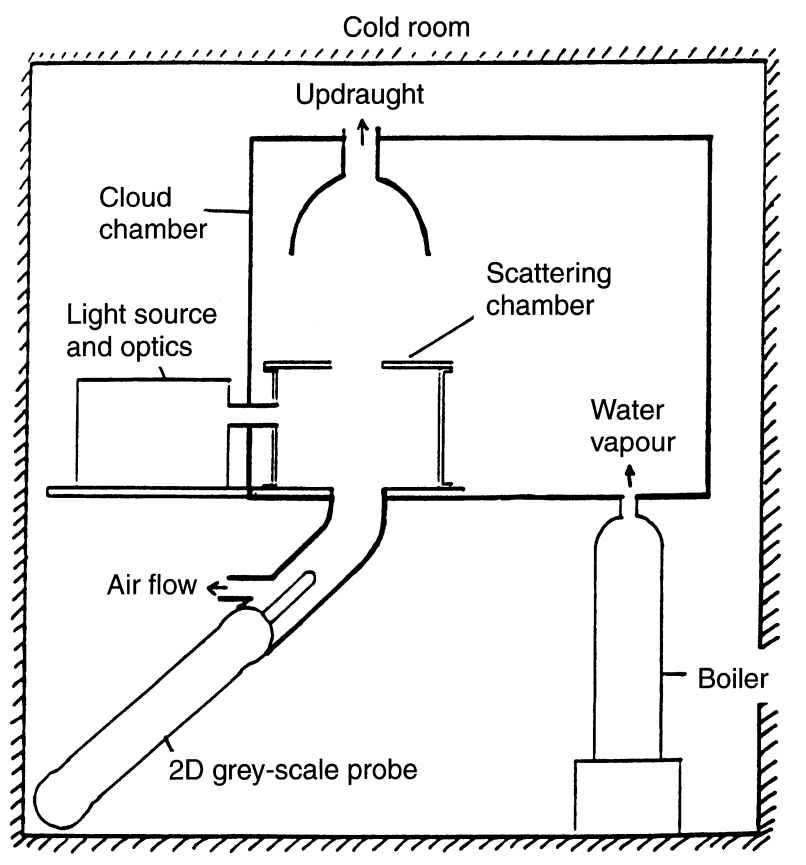

Fig. 1. The scattering chamber in the cold room together with the $2 \mathrm{D}$ ice crystal probe calculated the total projected area in order to determine the relative importance of the small versus the large crystals and found that small crystals can contribute significantly to the total area. Thus the laboratory generated single ice crystals used in the present work can represent the natural size distribution. The small crystals in the laboratory will have random orientations, which is representative of such crystals in nature as evidenced by the $22^{\circ}$ halo. The largest crystals observed by Arnott et al. (1994) were column rosettes; these have been observed in the cloud chamber, though, their concentration is too low to enable scattering studies to be made.

Two main methods are available at UMIST for the measurement of ice crystal sizes and concentrations in the laboratory cold rooms, they are the formvar replication technique and the $2 \mathrm{D}$ grey-scale cloud probe. The formvar replication technique has been described by Hallett (1976a, b) and Saunders and Wahab (1973). The technique involves coating a microscope slide with a 3 or $4 \%$ solution of formvar (polyvinyl formal) dissolved in chloroform and allowing ice crystals or water drops to hit and stick to the slide. The slide is dried initially at low temperature so that only the chloroform evaporates, allowing the formvar to harden around the particle so that a replica of the particle is left in the plastic. The continuous formvar replicator develops this idea further and gives a time-record of the cloud particles which are drawn past a moving film, recently coated with formvar solution, the particles stick and the formvar is dried before reaching the film take-up reel. If the particles pass the film with a known velocity and collection efficiency, a record of particle concentration with time can be produced. The continuous replicator is generally difficult to use and requires thorough cleaning with chloroform after use, to avoid clogging up with dried formvar. Data reduction is time consuming and tedious, requiring visual counting and sizing using a microscope.

The $2 \mathrm{D}$ probe is an optical particle sizing device (Knollenberg, 1970, 1976), which can be used to give real-time particle size and concentration data. The 2D Grey Probe consists of a He-Ne laser which is used to illuminate an array of 64 photodiodes. Cloud particles passing through the laser beam cast a shadow on the array. The crystals are imaged with a minimum detectable size of $10 \mu \mathrm{m}$ and the images are corrected for depth of field (a function of particle size) and array edge effects, and are stored in a computer file. If the velocity of the air flow which carries the particles through the probe is known, the particle concentration can be determined along with the size distribution. These data correlate directly with the actual sample of cloud on which the scattering measurements are performed so that optical and microphysical properties can be closely related in real time. Furthermore, the probe images can be used to determine an average projected area of particles in a given size range. The ice crystals were falling steadily in the scattering chamber without any unrealistic imposed orientation because the airflow rate through the probe (15 cm diameter) was only $0.3 \mathrm{~m} \mathrm{~s}^{-1}$ and the laser beam in the scattering chamber (diameter 
$45 \mathrm{~cm}$ ) was $15 \mathrm{~cm}$ above the port to the $2 \mathrm{D}$ probe, so there was little chance of the crystals becoming orientated in the chamber.

In general, a polar nephelometer is used to measure the scattering properties of ice and water clouds. The instrument normally consists of a collimated or focused light source aimed through a region where the cloud is allowed to form. A detector is used to measure the intensity of the scattered light at scattering angles between $0^{\circ}$ and $180^{\circ}$ and polarisers can be placed over the source and receiver to allow polarisation measurements to be made. Alternatively two detectors may be used to measure the horizontal and vertical polarisations simultaneously as employed by Sassen and Liou (1979a, b). A transmissometer measures the transmission properties of particulate clouds. The reduction in intensity as parallel light rays pass through a scattering medium is caused by absorption in the medium as well as scattering of radiation by the medium. The measured sum of these two effects is extinction. For wavelengths in the visible it is possible to derive extinction from angular scattering measurements by assuming that there is no light absorbed by the particle, which is very nearly true.

An instrument capable of making scattering and transmission measurements in a laboratory-produced ice crystal cloud has been developed and is shown in Fig. 2. A helium-neon laser $(0.63 \mu \mathrm{m})$ is used as the light source and solid-state detectors mounted on a rotating table determine the direct and scattered radiation from the ice crystals which are drawn through the scattering chamber to be sized by the $2 \mathrm{D}$ grey probe. The table is rotated from $10^{\circ}$ to $170^{\circ}$ in about $30 \mathrm{~s}$ under computer control using a stepper motor which provides an angular resolution of just under $0.5^{\circ}$. The back-scattered light cannot be determined since the detector must pass in front of the source beam and therefore obstructs it. In the forward direction, the detector saturates when the straight through position is approached, given the sensitivity required to detect light scattered laterally. The laser used as the light source has a minimum power output of $5 \mathrm{~mW}$ and is polarised to greater than 500:1. A spatial filter is used with a $\times 5$ or $\times 12$ beam expander to give a 4 or $10 \mathrm{~mm}$ collimated beam, respectively. The major design considerations of the solid-state detector heads are size, shielding requirements, sensitivity and the requirement for use in the cold room under adverse conditions which involves sealing to avoid the ingress of

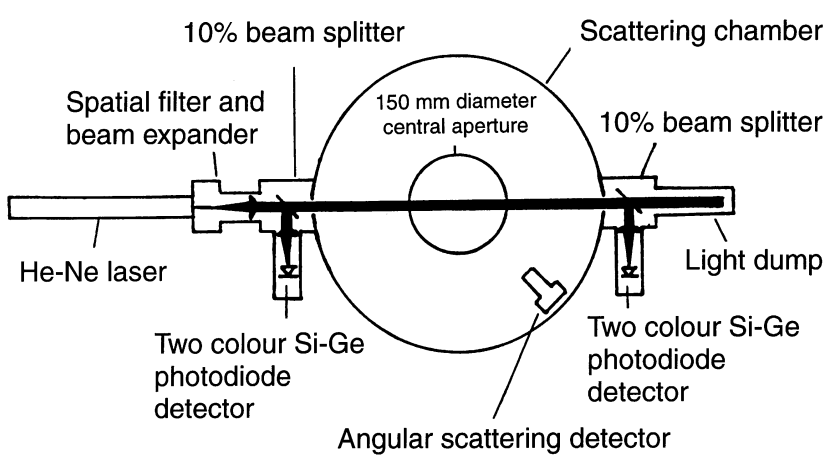

Fig. 2. Plan view of the scattering and extinction instrumentation water. Detectors are silicon photodiodes suitable for measurements up to a wavelength of $1 \mu \mathrm{m}$. Two GlanThompson Polarisation Prisms permit scattered light polarisation measurements. At present, the first polariser prism passes light at $45^{\circ}$ to the vertical and the second is in the same plane; in future measurements, the laser itself will be rotated to produce vertical or horizontally polarised beams that will allow the determination of the direct and depolarised components of the scattered radiation.

\section{Preliminary results and discussion}

Figure 3 shows some unnormalised scattering measurements following the seeding of a cloud of supercooled water droplets at $-7{ }^{\circ} \mathrm{C}$. The initial trace is for an allwater cloud. As time progresses the mixed column and plate ice crystals, of maximum dimension $50 \mu \mathrm{m}$, give enhanced scattering at $90^{\circ}$ relative to the initial water cloud. The reduction in scattered intensity in the later scans is due to the dissipation of the water cloud and ice crystal depletion when the crystals fall out of the cloud.

Extinction measurements can be made with the entry and exit port detectors. The first $100 \mathrm{~s}$ of Fig. 4 show a growing water cloud at $-15^{\circ} \mathrm{C}$, the cloud source was then turned off and at $280 \mathrm{~s}$ the cloud was nucleated. Measured angular scattering intensities can be made independent of particle concentration by dividing them by the simultaneously measured beam extinction. The second curve in the figure shows that when the scattered intensity at $112^{\circ}$ is divided by extinction, the result is almost independent of the widely varying water cloud extinction. However, when the ice crystals start to grow, the crystals scatter light at around this angle more effectively than do the water droplets.

The ice crystal cloud phase function depends on the particle concentration and on the projected crystal area. The example in Fig. 5 shows the crystal size distribution for hexagonal plate crystals and its shift to lower concentrations with time. Figure 6 is for the same data

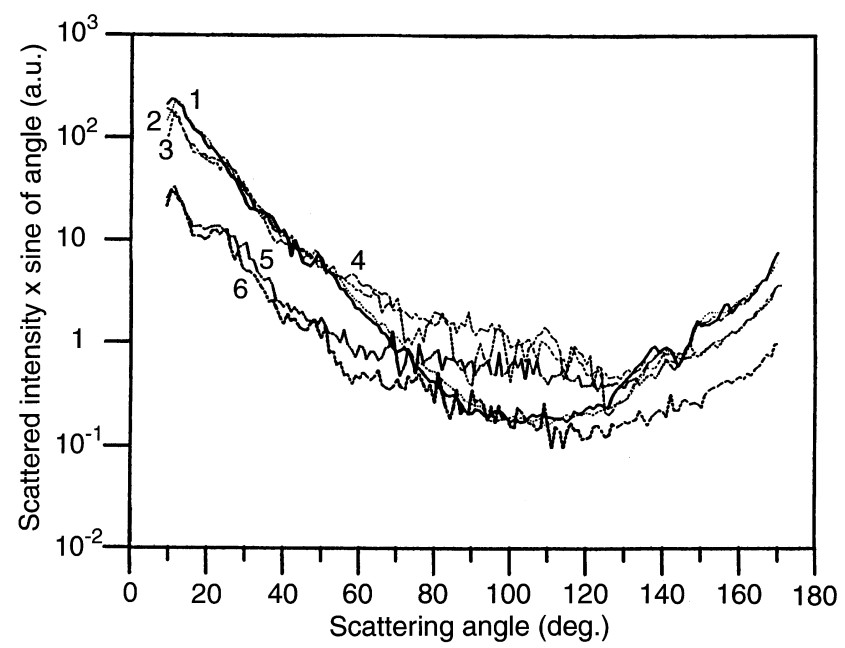

Fig. 3. The effect on the scattering of the life cycle of the ice crystal cloud in the chamber 


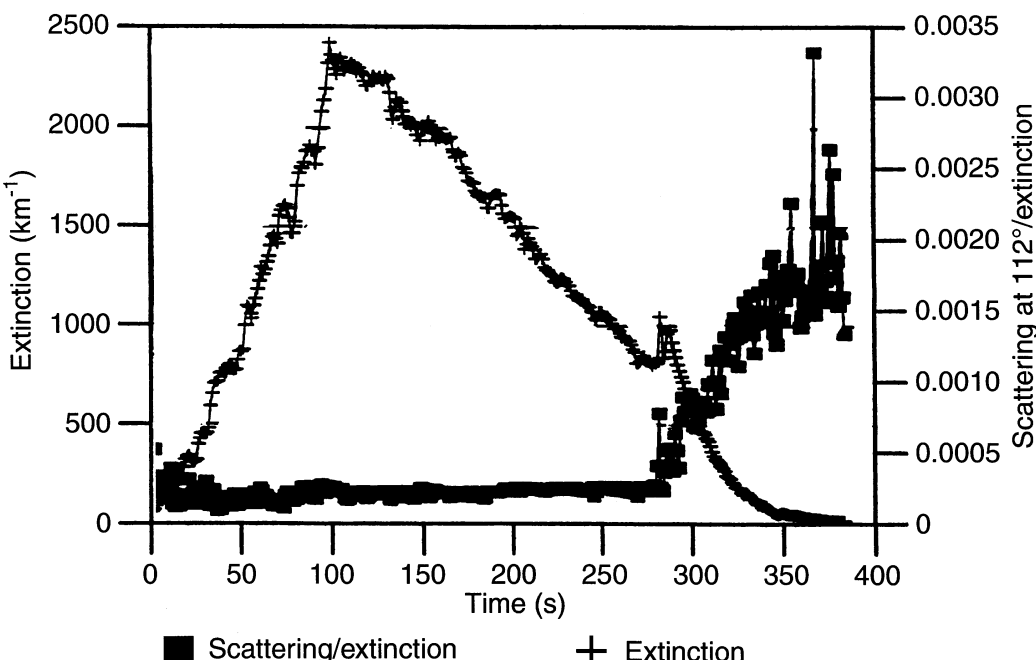

Fig. 4. Extinction and scattering/extinction at $112^{\circ}$ for a cloud nucleated at $280 \mathrm{~s}$

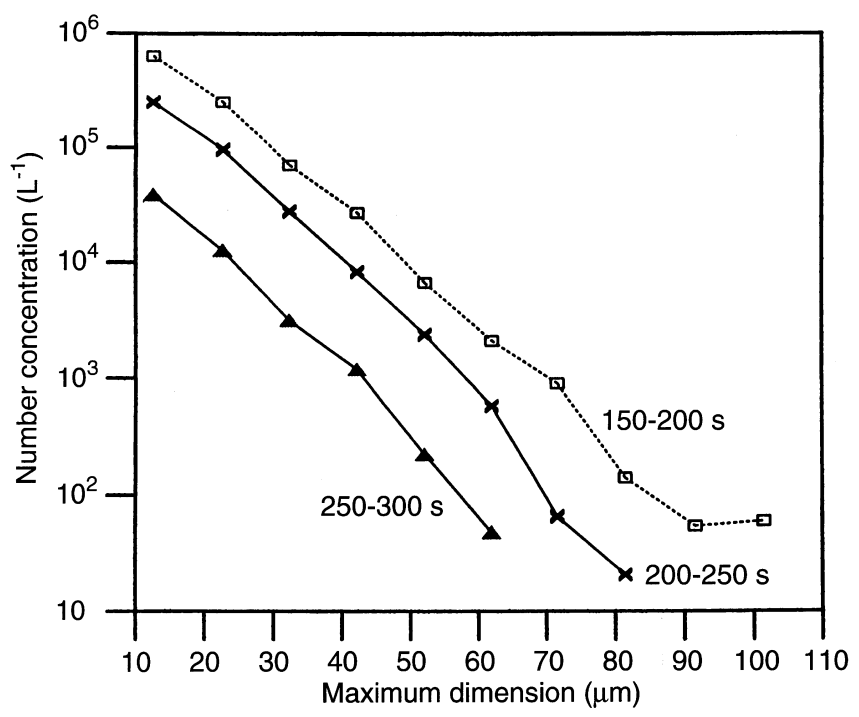

Fig. 5. Ice crystal size distributions for a dissipating cloud at $-15^{\circ} \mathrm{C}$

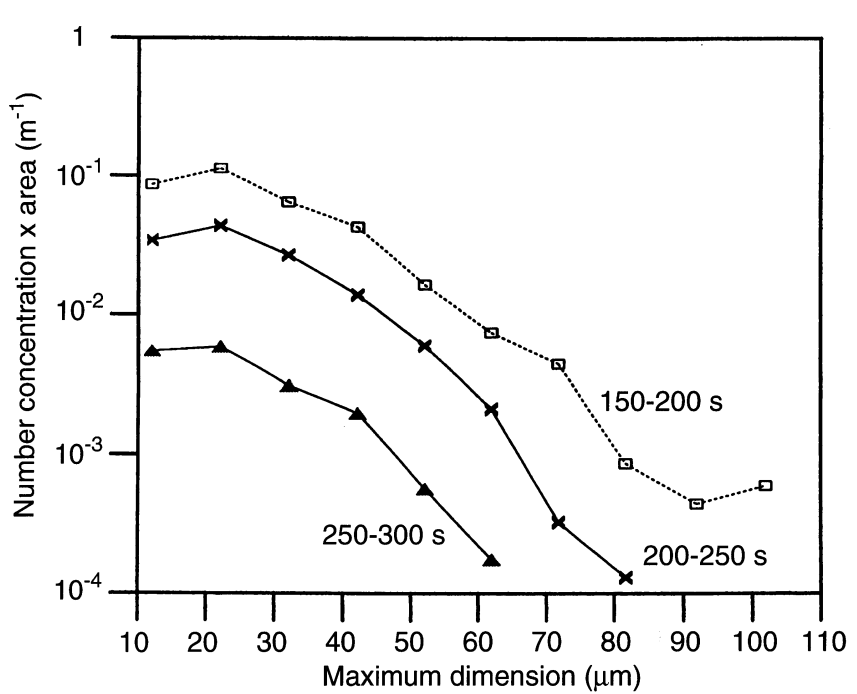

Fig. 6. Ice crystal number concentrations from Fig. 6 multiplied by the appropriate ice crystal area and shows the product of crystal concentration and corresponding crystal area. In this case the maximum effect on cloud extinction is caused by crystals of size about $20 \mu \mathrm{m}$. This ties in with the data of Heymsfield and Platt (1984), in which the cloud phase function is almost completely dominated by small crystals around $20 \mu \mathrm{m}$. This is a good example of the usefulness of the PMS probe. The important parameter for the optical cross-sections is the area projected by the crystals, which will undoubtedly have a different relationship to the maximum dimension for different crystal habits. We therefore have a method of directly determining the projected area which does not rely on models of idealised hexagonal structures.

A scattering phase function measured in the UMIST cold room with a cloud of hexagonal plate crystals of size around $20 \mu \mathrm{m}$ for an incident wavelength of $0.63 \mu \mathrm{m}$ is shown in Fig. 7 together with calculated values; the data have been normalised by taking account of the total extinction in the laser beam. Although the

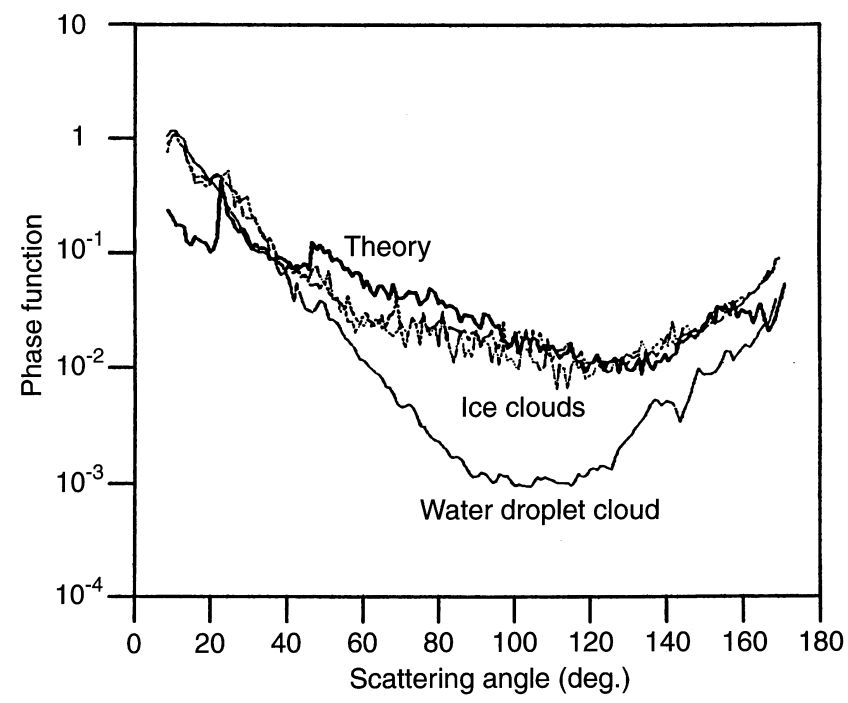

Fig. 7. Comparison between the measured and theoretical ice crystal scattering phase functions 

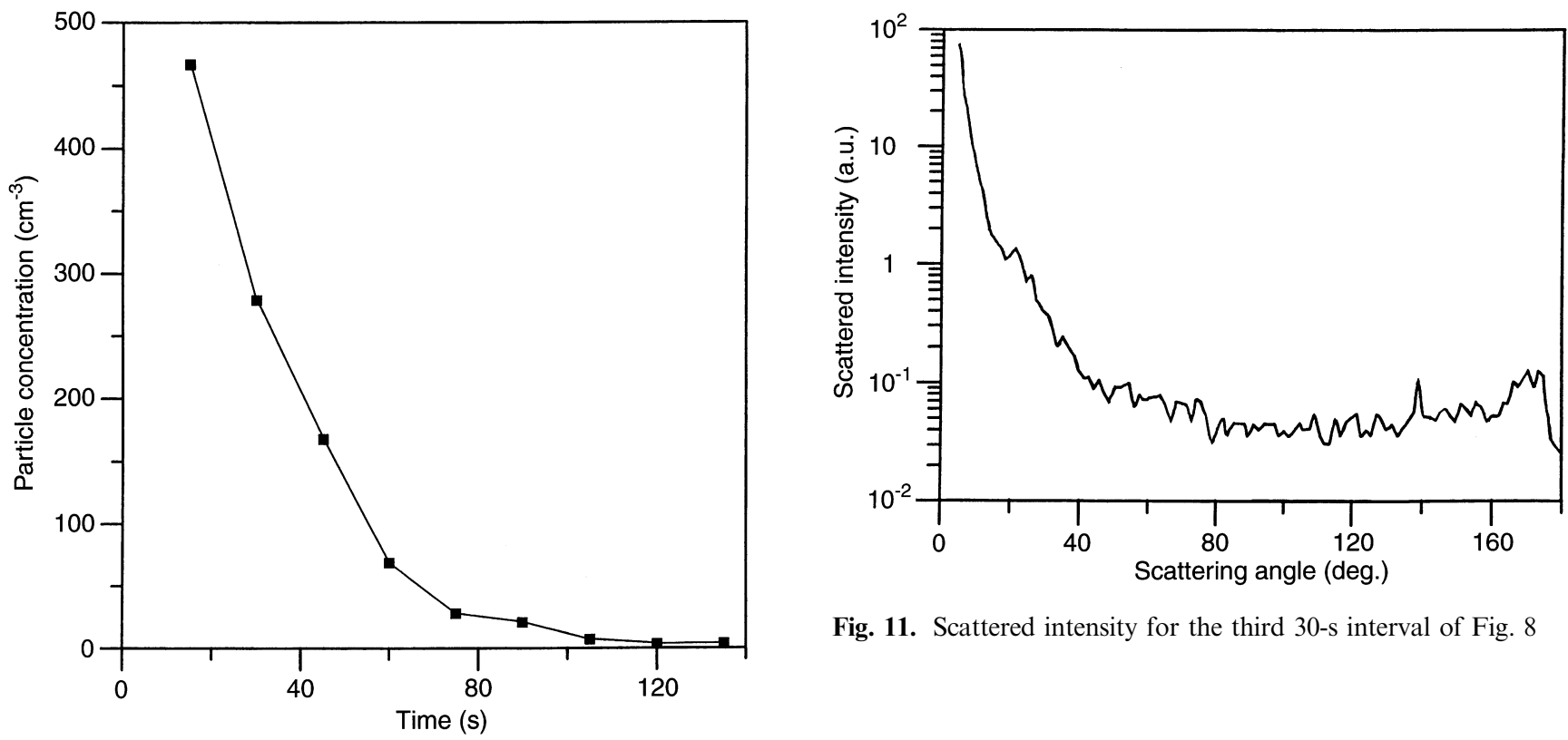

Fig. 11. Scattered intensity for the third 30 -s interval of Fig. 8

Fig. 8. Ice crystal concentration with time for a second cloud
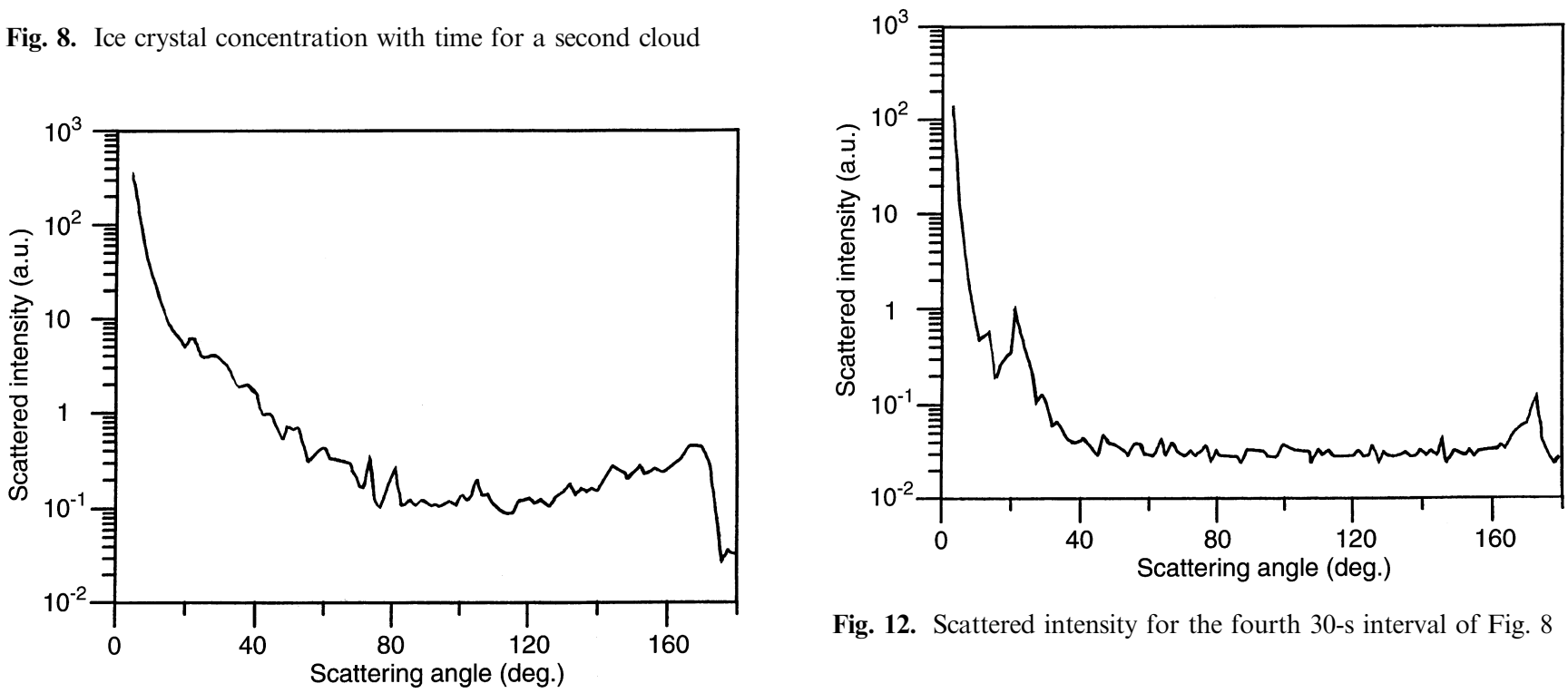

Fig. 12. Scattered intensity for the fourth 30 -s interval of Fig. 8

Fig. 9. Scattered intensity for the first 30 -s interval of Fig. 8

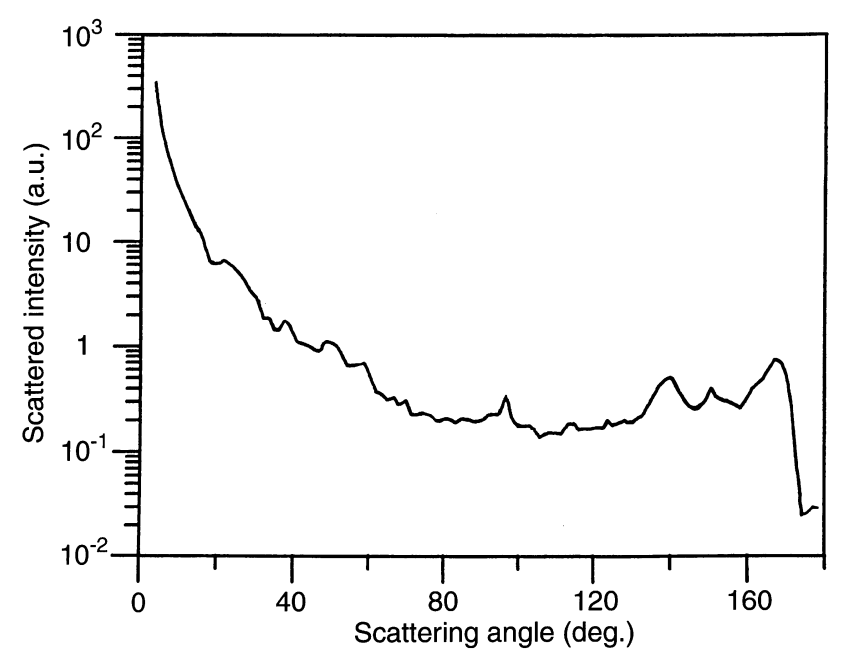

Fig. 10. Scattered intensity for the second 30-s interval of Fig. 8

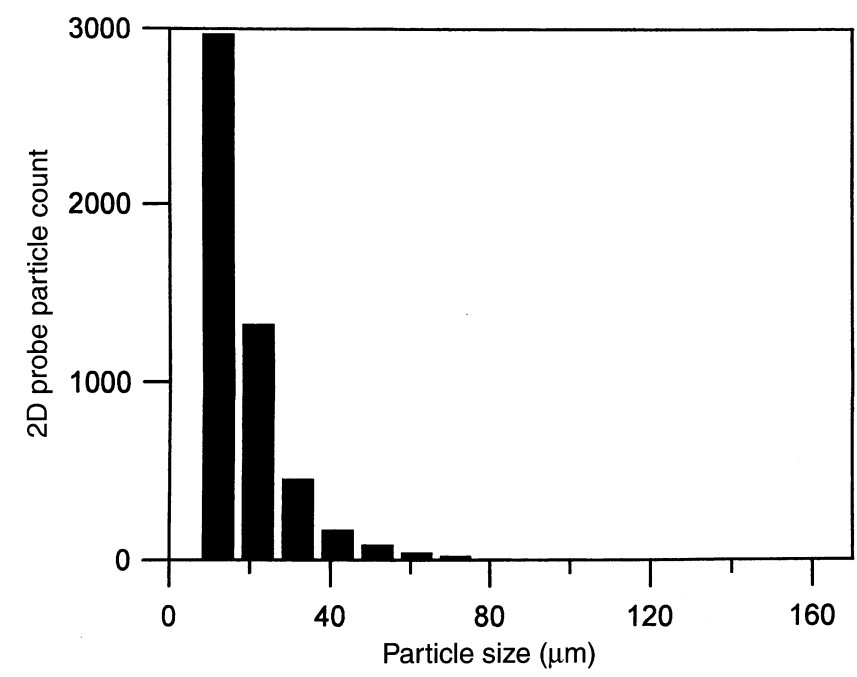

Fig. 13. Size distribution corresponding to Fig. 9 


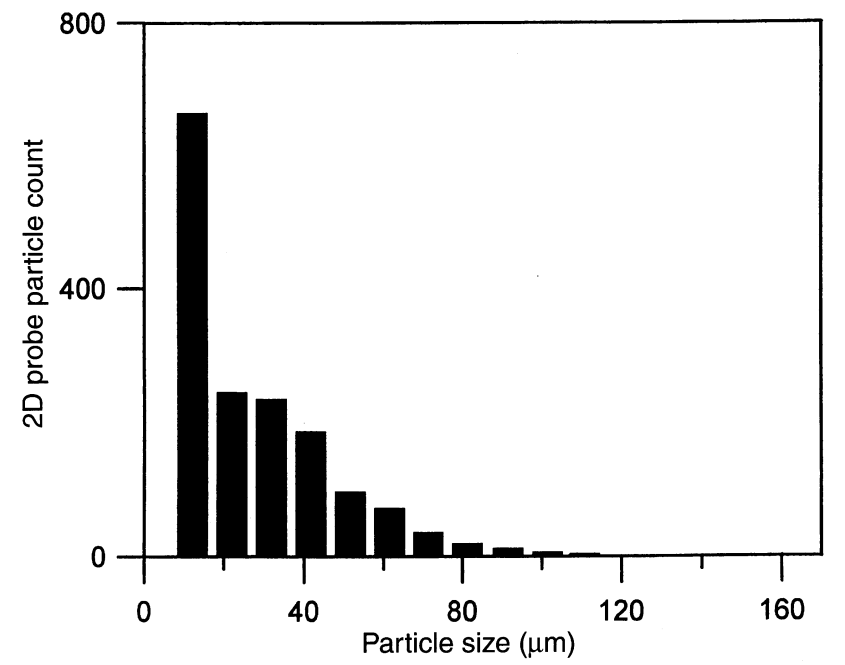

Fig. 14. Size distribution corresponding to Fig. 10

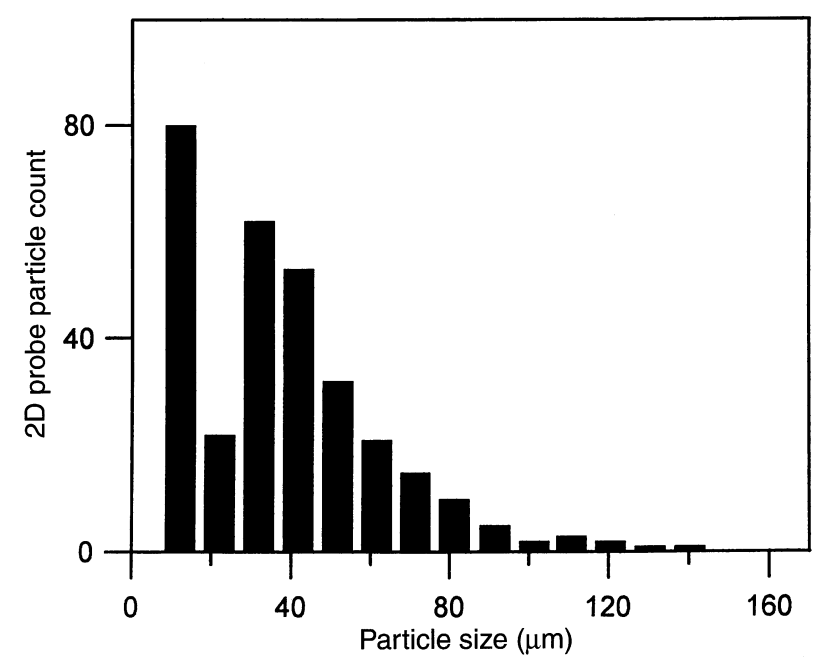

Fig. 15. Size distribution corresponding to Fig. 11

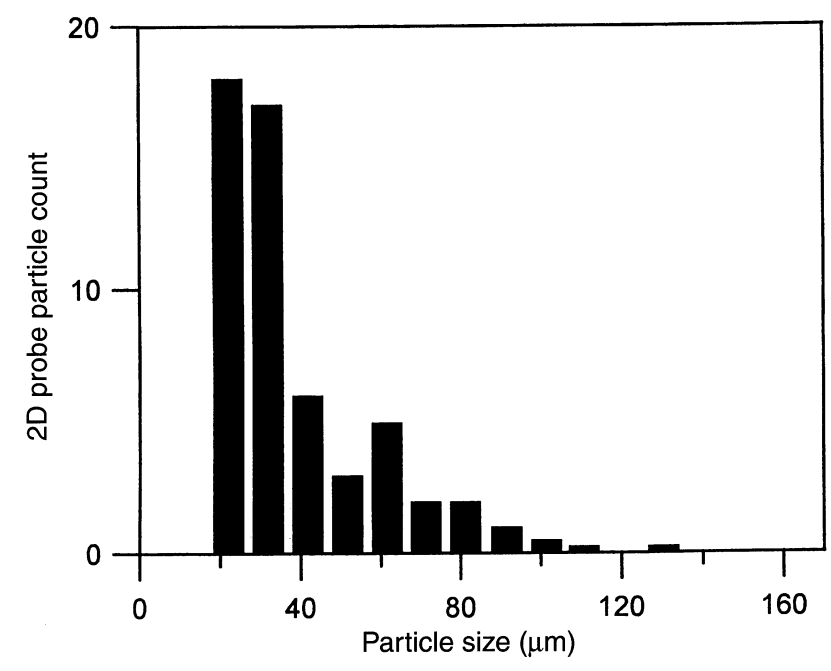

Fig. 16. Size distribution corresponding to Fig. 12 shapes are similar, no $22^{\circ}$ halo is observed possibly due to multiple scattering, particle surface roughness or an effect due to the many small crystals present.

If many small particles (dimension around $10 \mu \mathrm{m}$ ) exist in the laboratory cold room or real cirrus cloud, diffraction will dominate the single ice crystal scattering phase function angular distribution; the diffracted energy will be much more widely spread in the forward direction than for larger particles, so the slope of the scattering curve will be relatively flat around an angle of $<30^{\circ}$. Although the ray-tracing method is not applicable for very small particles $(D<20 \mu \mathrm{m})$, it can give a qualitative explanation. The model calculation for extremely small particles shows that the $22^{\circ}$ halo will be indistinguishable from the background scattering when the particle is very small.

Macke (1994) studied the multiple scattering phase function for a homogeneous ice crystal cloud and found that even for an optical thickness of 10 - an extremely large value for cirrus clouds - the $22^{\circ}$ halo is still detectable. Macke also studied the effect of crystal surface roughness and noted the removal of peaks in the phase function.

The laboratory results mentioned in the foregoing, in which the experiments did not produce a halo, have been repeated with a modified cloud nucleation system that permits the growth of a smaller concentration of crystals, thus avoiding problems of multiple scattering. Figure 8 shows the averaged crystal concentration with time in experiments in which the crystals grew at temperatures around $-15^{\circ} \mathrm{C}$; Figs. 9-12 show successive scattering results as the crystals grow and fall out of the cloud. The crystal size distributions averaged over each scan time are shown in Figs. 13-16, respectively. The later results verify the existence of the halo at $22^{\circ}$ while showing up the problem involved in loss of sensitivity at low light scattering levels when the ice crystal concentration is depleted.

Future plans are to use the apparatus to study the scattering properties of laboratory grown ice crystal clouds, in particular to examine the effect of ice crystal size and morphology. Presently, the wavelength of interest is in the visible, future work will involve a range of visible wavelengths of relevance to the effects of the solar spectrum. Later, longer wavelengths will be utilised to study the effects of cirrus ice crystal clouds on the scattering and absorption of terrestrial radiation.

\section{Conclusions}

A new laboratory-based ice crystal cloud facility has been described which will permit studies of the optical scattering effects of ice crystals representative of those in cirrus clouds. Comparisons between the laboratory results and theoretical studies will allow improvements to numerical models of the effects of cirrus clouds on the global climate.

A PMS grey-scale probe has been used to correlate the microphysical cloud properties in real time. The use of the probe in the laboratory is convenient since the 
particles responsible for the scattering are the same particles as are sampled by the probe. Also, the average projected area for a given maximum dimension can be directly measured which will allow us to determine the effect of different crystal sizes on cloud extinction.

Preliminary results show that the scattering effect of ice crystals at angles between $60^{\circ}$ and $140^{\circ}$ is considerably more important than is the case with a cloud of water droplets. We have observed the evolution of the scattering properties when ice crystals grown from a cloud of supercooled water droplets fall out in the cloud chamber, thus depleting the cloud. In particular, with lower cloud densities, the $22^{\circ}$ halo begins to appear when the ice crystals grow larger and multiple scattering is reduced when the particle concentration falls.

In future studies the effects of ice crystal size, shape and orientation will be examined together with polarisation effects; the wavelength range will be extended beyond the visible in studies of infrared absorption.

Acknowledgements. Topical Editor K.-H. Glaßmeier thanks A. Heymsfield and G. Brogniez for their help in evaluating this paper.

\section{References}

Arnott, W. P., Y. Y. Dong, J. H. Hallett, and M. R. Poellot, Role of small ice crystals in radiative properties of cirrus: a case study, FIRE II, November 22, 1991, J. Geophys. Res., 99, 1371-1381, 1994.

Brogniez, G., J. C. Buriez, V. Giraud, F. Parol, and C. Vanbauce, Determination of effective emittance and a radiatively equivalent microphysical model of cirrus from ground-based and satellite observations during the International Cirrus Experiment: the 18 October 1989 case study, Mon. Weather Rev., 123, 1025-1036, 1995.

Bunting, J. T., Sensing ice clouds from satellites, In: Light scattering by irregularly shaped particles, Ed, D.W. Schuerman, Plenum Press, New York, pp. 25-34, 1980.

Dowling, D. R., and L. F. Radke, A summary of the physical properties of cirrus clouds, J. Appl. Meteorol, 29, 970-978, 1990.

Downing, H. D., and D. Williams, Optical constants of water in the infrared, J. Geophys. Res., 80, 1656-1661, 1975.

Dugin, V. P., B. M. Golubitskiy, S. O. Mirumyants, P. I. Paramonov, and M. V. Tantashev, Optical properties of artificial ice clouds, Izv. Atmos. Ocean. Phys., 7, 871-7, 1971.

Dugin,V. P., O. A. Volkovitskiy, S. O. Mirumyants, and N. K. Nikiforova, Anisotropy of light scattering by artificial crystalline cloud formations, Izv. Atmos. Oceanic Phys., 13, 22-25, 1977.

Foot, J. S., Some observations of the optical properties of clouds. II: cirrus. Q. J. R. Meteoral. Soc., 114, 145-164, 1988.

Gayet, J. F., O. Crépel, J. F. Fournal, and S. Oschepkov, A new airborne polar nephelometer for the measurements of optical and microphysical cloud properties. Part 1: theoretical design, Ann. Geophysicae, 15, 451-459, 1997.

Hale, G. M., and M. R. Querry, Optical constants of water in the $200 \mathrm{~nm}$ to $200 \mu \mathrm{m}$ wavelength region, Appl. Opt, 12, 555-63, 1973.

Hallett, J., Cloud particle replicator for use on a pressurized aircraft: part 1. Operating Manual, Air Force Geophysics Laboratory, United States Air Force, Hanscom AFB, Mass., 1976a.

Hallett, J., Cloud particle replicator for use on a pressurized aircraft: part 2. Operating Manual, Air Force Geophysics Laboratory, United States Air Force, Hanscom AFB, Mass., 1976b.
Heymsfield, A. J., and C. M. R. Platt, A parameterization of the particle size spectrum of ice clouds in terms of the ambient temperature and the ice water content, J. Atmos. Sci., 41, 846$855,1984$.

Hobbs, P. V., Ice physics, Clarendon Press, Oxford, 1974.

Huffman, P. J., Polarization of light scattered by ice crystals, J. Atmos. Sci., 27, 1207-8, 1970.

Huffman, P. J., and W. R., Thursby Jr., Light scattering by ice crystals, J. Atmos Sci., 26, 1073-1077, 1969.

Jacobowitz, H., Emission, scattering and absorption of radiation in cirrus cloud layers, $\mathrm{PhD}$ thesis, Department of Meteorology, MIT, 1970.

Kinne, S. A., Parameterization of radiative transfer in the earth's atmosphere with specific application to clouds. PhD thesis University of Utah, 1987.

Kinne, S. A., T. P. Ackerman, A. J. Heymsfield, F. P. J. Valero, K. Sassen, and J. D. Spinhirne, Cloud microphysics and radiative transfer: cloud field study on 28 October 1986, Month. Weather Rev., 120, 661-684, 1992.

Knollenberg, R. G., The optical array: an alternative to scattering or, extinction for airborne particle size determination, J. Appl. Meteorol, 9, 86-103, 1970

Knollenberg, R. G., Three new instruments for cloud physics measurement: the 2-D spectrometer, the forward scattering spectrometer probe and the active scattering spectrometer probe and the active scattering aerosol spectrometer, Preprints Int. Conf. on Cloud Physics, Boulder, 544-561, 1976.

Liou, K. N., An introduction to atmospheric radiation, Academic Press, New York, 1980.

Liou, K. N., Some aspects of the optical properties of ice clouds, In: Clouds, their formation, optical properties and effects, Eds. P. V. Hobbs and A. Deepak, Academic Press, New York, pp. 315359, 1981.

Liou, K.N., R. Baldwin, and T. Kaser, Preliminary experiments on the scattering of polarized light by ice crystals, J. Atmos. Sci., 33, 553-557, 1976.

Liu, C., P. R. Jonas, and C. P. R. Saunders, Phase function calculations of single hexagonal bullet-like ice crystals, Ann. Geophysicae, 13, 1348-1354, 1995.

Liu, C., P. R. Jonas, and C. P. R. Saunders, Accuracy of the anomalous diffraction approximation to light scattering by column-like ice crystals, Atmos Res., 41, 63-69, 1996a.

Liu, C., P. R. Jonas, and C. P. R. Saunders, Pyramidal ice crystals scattering phase functions and concentric halos, Ann. Geophysicae, 14, 1192-1197, 1996b.

Macke, A., Scattering of light by irregular ice crystals in threedimensional inhomogeneous cirrus clouds. Proc. 8th Conference on Atmospheric Radiation. Pub. Am. Meteorol. Soc., pp 304-306, 1994

Magono, C., and C. W Lee, Meteorological classification of natural snow crystals, J. Fac. Sci. Hokkaido Univ., Ser. 7, 2, 321-335, 1966.

Mason, B.J., The physics of clouds, Clarendon Press, Oxford, 1971.

Mischenko, M. I., W. B. Rossow, A. Macke, and A. A. Lacis, Sensitivity of cirrus cloud albedo, bidirectional reflectance and optical thickness retrieval accuracy to ice particle shape, J. Geophys. Res., 101, 16 973-16 985, 1996.

Monteith, J. L., and M. H. Unsworth, Principles of environmental physics, Edward Arnold, 36-57, 1990.

Paltridge, G. W., and C. M. R. Platt, Aircraft measurements of solar and infrared radiation and the microphysics of cirrus clouds. Q. J. R. Meteorol. Soc. 107, 367-380, 1981.

Platt, C. M. R., J. D. Spinhirne, and W. D. Hart, Optical and microphysical properties of a cold cirrus cloud: evidence for regions of small ice particles, J Geophys. Res., 94, 11 151$11164,1989$.

Pluchino, A., Scattering photometer for measuring single ice crystals and evaporation and condensation rates of liquid drops, J. Opt. Soc. Am. A, 4, 614-620, 1987.

Sassen, K., and K. N. Liou, Scattering of polarized laser light by water droplet, mixed phase and ice crystal clouds. Part I: 
angular scattering patterns, J. Atmos. Sci., 36, 838-851, 1979a.

Sassen, K., and K. N. Liou, Scattering of polarized laser light by water droplet, mixed phase and ice crystal clouds. Part II: angular depolarizing and multiple scattering, J. Atmos. Sci., 36, 852-861, 1979b.

Saunders, C. P. R., and N. A. Wahab, The replication of ice crystals, J. Appl. Meterol., 12, 1035-1039, 1973.
Takano,Y., and K. N. Liou, Solar radiative transfer in cirrus clouds. Part I: single scattering and optical properties of hexagonal ice crystals, J. Atmos. Sci., 46, 3-19, 1989a.

Takano,Y., and K. N. Liou, Solar radiative transfer in cirrus clouds. Part II: theory and computation of multiple scattering in anisotropic medium, J. Atmos. Sci., 46, 20-36, 1989b.

Warren, S. G., Optical constants of ice from the ultraviolet to the microwave, Appl. Opt., 23, 1206-1225, 1984. 